Gefässchirurgie 2014 • 19:102-103

DOI 10.1007/s00772-013-1250-5

Online publiziert: 21. März 2014

(c) Springer-Verlag Berlin Heidelberg 2014

E.S. Debus ${ }^{1} \cdot$ M. Augustin ${ }^{2}$

${ }^{1}$ Klinik und Poliklinik für Gefäßmedizin, Universitäres Herzzentrum Hamburg, Universitätsklinikum Hamburg-Eppendorf, Hamburg

${ }^{2}$ Institut für Versorgungsforschung in der Dermatologie und bei Pflegeberufen (IVDP), Universitätsklinikum Hamburg-Eppendorf, Hamburg

\title{
PAVK - eine der wichtigsten Herausforderungen der vaskulären Medizin
}

lich zur Verfügung zu stellen. Der Gesetzgeber macht es in Deutschland der Industrie im Vergleich zu vielen anderen industrialisierten Ländern erstaunlich leicht: Die Vergabe eines CE-Labels durch sog. „benannte Stellen“ stellt keine entscheidende Hürde für die Zulassung dar. Anwendungsbeobachtungen reichen aus, um für den stationären Einsatz zugelassen zu werden. Nach den Kriterien der evidenzbasierten Medizin entspricht dies dem niedrigsten Evidenzniveau. Das ist bemerkenswert - und macht Deutschland sowohl für die forschende Medizinindustrie wie für uns Anwender gleichermaBen als Wissenschaftsstandort attraktiv. Wer möchte nicht zu den ersten zählen, die (möglichst gute) Daten über eine neue Technik oder ein neues Produkt publiziert haben?

\section{》) Die vaskuläre Medizin ist von Fortschritten in der Pharmakotherapie und der Medizinproduktentwicklung gekennzeichnet}

Reichen diese Vorgaben aber aus, um verantwortlich am Patienten eingesetzt zu werden? Wir müssen nicht weit zurückschauen, um auf Beispiele für den verfrühten (weil eben nicht ausreichend evidenzbasierten) Einsatz neuer Technologien mit verhängnisvollen Folgen für den Patienten zu stoßen - man denke nur an die ersten Generationen aortaler Stentgraftsysteme vor 20 Jahren, die nahezu alle wieder explantiert werden mussten.
Aus verschiedenen Erhebungen (s. auch Beiträge von Eckstein et al. und Debus et al. in diesem Heft) wissen wir recht gut, welche Therapien in Deutschland wie häufig und von wem eingesetzt werden. Die Gefäßchirurgie spielt in Deutschland bei der Therapie der PAVK eine zentrale Rolle, aber wissen wir, wie gut unsere Behandlungen im Langzeitverlauf und quo ad vitam wirklich sind? Daten des Statistischen Bundesamtes weisen eindrücklich eine seit vielen Jahren kontinuierlich rückläufige Mortalität nach, und auch die Notwendigkeit einer langwierigen stationären Behandlungen der PAVK sinkt. Dazu fallen die Majoramputationsraten signifikant bei gegenläufigen Minoramputationen - ein aussagekräftiger Indikator für die Therapieeffektivität. Parallel dazu steigt die Zahl von Fachärzten und eigenständigen gefäßchirurgisch spezialisierten Einrichtungen kontinuierlich an. Ein Zusammenhang liegt nahe.

Die Frage aber, ob und wann wir welche Therapie in welchem Stadium einsetzen sollten, basiert nach Evidenzkriterien auf einer vergleichsweise dünnen Datenlage. Es ist nicht erstaunlich, dass die Medizinprodukteindustrie die Evaluierung eigener Neuentwicklungen im Auge hat. Der Vergleich mit einer offen-chirurgischen Maßnahme spielt in den aktuellen (vornehmlich industriegesponsorten) Untersuchungen zur invasiven Therapie der PAVK keine Rolle - er kommt nicht mehr vor. Das ist bedenklich. Die vorliegenden Studien sind hinsichtlich ihrer Endpunkte, Designs und 
Studiengruppen darüber hinaus so uneinheitlich, dass auch eine Zusammenfassung im Sinne von aussagekräftigen Metaanalyen nicht möglich ist. Die Generierung externer Evidenz zum Einsatz medizintechnischer Innovationen ist teuer, keine Frage, sie wäre aber machbar. Die derzeitige Praxis einer ausschließlichen Industriefinanzierung von (Zulassungs-) Studien sollte überdacht werden. Es sollte überlegt werden, ob eine Umverteilung auf den Gesetzgeber und die Kostenträger nicht sinnvoller wäre.

Im Vordergrund der Bewertung von medizintechnischen Innovationen muss auch künftig die Patientensicherheit stehen, die nicht zugunsten von ökonomischen Partialinteressen aufgegeben werden darf.

Eine Alternative zu randomisierten klinischen Studien stellen populationsbasierte Register dar, die für die Karotisendarteriektomie und das abdominelle Aortenaneurysma in Deutschland gut etabliert, aber für die PAVK noch Neuland sind. Sie haben für die Therapieentscheidungen der Karotisstenose und des Aortenaneurysmas eine hohe Evidenz generiert - für die PAVK steht sie noch aus. Die Erfahrungen der vergangenen Jahre zeigt aber, dass wir eine Registratur für die Therapie der pAVK brauchen werden. Studien haben sie bislang nicht hergegeben. Ein Blick ins Ausland zeigt darüber hinaus: Trotz hohen Aufwandes für jeden Einzelnen ist dies möglich. Wir haben im Vorstand der DGG daher in einem Pilotprojekt einen ersten Schritt in diese Richtung unternommen und orientieren uns in der Umsetzung an den Erfahrungen benachbarter Länder, wo (wie u.a. in Schweden, Finnland, der Schweiz oder Großbritannien) teilweise eine jahrzehntelange Erfahrung besteht.

Erste Auswertungen aus diesem regionalen Register werden wir auf unserer Frühjahrstagung vorstellen, zu der Sie auf diesem Wege herzlich eingeladen sind! Sie findet am 27.-28.3.2014 im Rahmen der 131. Jahrestagung der DGCh in Berlin unter das Motto „Gefäßchirurgie zwischen Faszination, Mut und Demut" statt, mit der wir eine neue weiterbildungsorientierte Plattform geschaffen haben, die sowohl für „alte Hasen“ als auch für wissbegierige Nachwuchsspezialisten eine ideale Möglichkeit zur zielgerichteten Weiterbildung ist. Besonders möchte ich auf unser neues GPS-Programm (Gefäßmedizin BerufsPolitik Standortbestimmungen) hinweisen, welches ein hochspannendes berufspolitisches Diskussionsforum darstellt, auf welchem die o.a. Themen und weitere Hot Topics mit renommierten Rednern aus Politik und Selbstverwaltung diskutiert werden.

Die PAVK wird weiter ein Thema bleiben. Hierzu planen wir die Produktion eines Imagefilms und einer AwarenessKampagne, um die frühzeitige Erkennung dieser wichtigen Markererkrankung, deren Prävention und Therapiemöglichkeiten bei Patienten und Kollegen in den Fokus zu rücken. Wir produzieren derzeit einen Ärzteinformationsflyer, der den ärztlichen Kreisverbänden bundesweit zugestellt werden wird, um auch lokal in Ärztefortbildungen gezielt informieren zu können. Hierzu sind Vortragsvorlagen als Orientierungsgrundlage erstellt worden, die jedem DGG-Mitglied vor Ort zur Verfügung stehen, sofern erwünscht.

Schließlich möchte ich Sie bereits heute auf unseren Jahreskongress vom 24.27.09.2014 im Hamburger CongressCentrum hinweisen, mit dem wir unter dem Motto „Einheit und Vielfalt" auch unser 30-jähriges Gesellschaftsjubiläum begehen. Die Vorbereitungen sind weit gediehen. Besuchen Sie unsere Homepage für weitere Informationen! $\mathrm{Zu}$ den Highlights werden u.a. unser Schlagabtausch, eine transatlantische Debatte (gemeinsam mit dem neu gegründeten German Speaking Chapter der SVS), eine europäische Sitzung, die Jörg-Vollmar-Lecture, die Vergabe von Stipendien und Preisen und vieles mehr zählen. Auch werden wir wieder ein breites Kursangebot im Vorfeld des Kongresses anbieten, welches im European Surgical Institute stattfinden wird. Das Kongressteam und ich freuen uns schon sehr, Sie im September in Hamburg begrüßen zu können!

Die PAVK bleibt eine der wichtigsten Herausforderungen der vaskulären Medizin. Wir wünschen Ihnen einen großen Erkenntnisgewinn bei der Lektüre dieses Heftes!
Herzlichst, Ihre

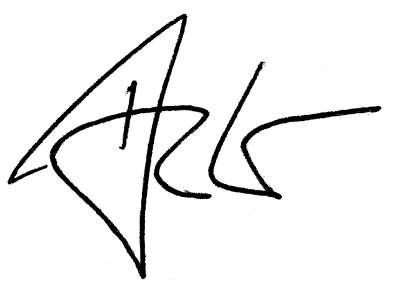

Prof. Dr. E. Sebastian Debus

Präsident der DGG 2013/2014

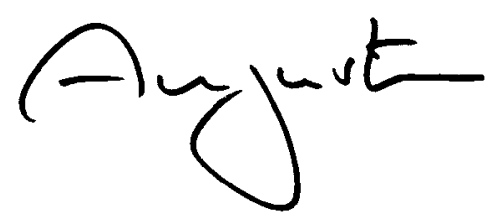

Prof. Dr. M. Augustin

Direktor IVDP, UKE Hamburg-

Eppendorf

\section{Korrespondenzadresse}

Prof. Dr. E.S. Debus

Klinik und Poliklinik für Gefäßmedizin, Universitäres Herzzentrum Hamburg, Universitätsklinikum Hamburg-Eppendorf Martinistraße 52

20246 Hamburg

s.debus@uke.de

\section{Prof. Dr. M. Augustin}

Institut für Versorgungsforschung in der Dermatologie und bei Pflegeberufen (IVDP),

Universitätsklinikum Hamburg-Eppendorf Martinistraße 52

20246 Hamburg

m.augustin@uke.de

Interessenkonflikt. E. S. Debus und M. Augustin geben an, dass kein Interessenkonflikt besteht. 